\title{
Effects of metformin and Exenatide on insulin resistance and AMPKa-SIRT1 molecular pathway in PCOS rats
}

\author{
Xin Tao ${ }^{1 *}$, Lisi Cai ${ }^{2}$, Lei Chen ${ }^{3}$, Shuqi Ge ${ }^{1}$ and Xuanying Deng ${ }^{1}$
}

\begin{abstract}
Aims: This study was designed to evaluate the protective effects of AMPKa and SIRT1 on insulin resistance in PCOS rats, and to illuminate the underlying mechanisms.

Methods: An in vitro PCOS model was established by DHEA $(6 \mathrm{mg} /(100 \mathrm{~g} \cdot \mathrm{d})$ ), and the rats were randomly divided into the metformin group (MF group, $n=11$ ), the exenatide group (EX group, $n=11)$, the PCOS group $(n=10)$, and the normal control group (NC group, $n=10$ ). The MF group was administered MF $300 \mathrm{mg} /(\mathrm{kg} \cdot \mathrm{d})$ daily. The EX group was subcutaneously injected EX $10 \mu \mathrm{g} /(\mathrm{kg} \cdot \mathrm{d})$ daily. After 4 weeks of continuous administration, fasting blood glucose and serum androgen, luteinizing hormone and other biochemical indicators were measured. Western and Real-time PCR were used to determine the expression of AMPKa and SIRT1 in the ovaries of each group.

Results: After 4 weeks of drug intervention, compared with untreated PCOS group, EX group and MF group had visibly decreased body weight $(222.64 \pm 16.57,218.63 \pm 13.18$ vs $238.30 \pm 12.26 \mathrm{~g}, P=0.026)$, fasting blood glucose $(7.71 \pm 0.72,8.17 \pm 0.54$ vs $8.68 \pm 0.47 \mathrm{mmol} / \mathrm{L}, P<0.01)$, HOMA-IR $(8.26 \pm 2.50,7.44 \pm 1.23$ vs $12.66 \pm 1.44, P<0.01)$ and serum androgen $(0.09 \pm 0.03,0.09 \pm 0.03$ vs $0.53 \pm 0.41 \mathrm{ng} / \mathrm{ml}, P<0.01)$ and the expressions of AMPKa and SIRT11 were increased progressively $(P<0.05)$.

Conclusions: Both metformin and exenatide can improve the reproductive and endocrine functions of rats with PCOS via the AMPKa-SIRT1 pathway, which may be the molecular mechanism for IR in PCOS and could possibly serve as a therapeutic target.
\end{abstract}

Keywords: Polycystic ovary syndrome, Obesity, Insulin resistance, AMPKa, SIRT1

\section{Introduction}

The polycystic ovary syndrome (PCOS) is a common endocrine disorder in women of reproductive age with a prevalence of $5-10 \%$ [1]. The syndrome is characterized by hyperandrogenism, ovulatory dysfunction and polycystic ovaries. PCOS, a syndrome of unknown etiology, is furthermore associated with accumulation of abdominal fat, obesity $\left(\mathrm{BMI}>30 \mathrm{~kg} / \mathrm{m}^{2}\right)$ and insulin resistance (IR), which are present in $70-80 \%$ of women of PCOS [2]. There is increasing global data linking PCOS to metabolic complications, such as impaired glucose tolerance (IGT), type 2 diabetes (DM2), dyslipidemia,

\footnotetext{
* Correspondence: doctort@163.com

${ }^{1}$ Center for Reproductive Medicine, The Third Affiliated Hospital, Sun Yat-sen University, 600, Thianhe Road, Guangzhou, Guangdong, People's Republic of China 510630

Full list of author information is available at the end of the article
}

elevated cardiovascular risk factors [3]. Due to the high incidence of obesity and IR in PCOS patients, weight reduction and lifestyle modification have become an important component in the treatment of the disease. However, many patients fail to lose weight or quickly regain fat. Effective intervention is urgently needed to minimize metabolic complications in patients with PCOS.

Insulin sensitizers, especially metformin (MF), have been shown as a pharmaceutical option aiming at not only IR, but also several other aspects of PCOS, including reproductive dysfunctions [4]. In 1994, Velazquez reported for the first time that MF had beneficial effects on reproductive as well as metabolic abnormalities in women with PCOS [5]. Since then, a lot of studies have confirmed the protective impact of MF on IR and 
obesity in women with PCOS. MF lowers blood glucose and enhances insulin sensitivity by reducing hepatic gluconeogenesis via activating AMP-activated protein kinase (AMPK) pathway [6]. A major limitation of its use is its side effects, which are predominantly gastrointestinal reactions consisting of nausea, diarrhea and bloating. Moreover, The weight loss effect of MF on the basis of lifestyle therapy does not seem to be very satisfactory [7].

Glucagon-like peptide 1 (GLP-1) is an incretin hormone that was primarily described in the 1980s as a proglucagon cleavage products, produced by intestinal cells in response to food intake [8]. It lowers postprandial glucose levels by promoting glucose-dependent insulin secretion, inhibiting glucagon secretion, decelerating the emptying of gastric contents and improving pancreatic $\beta$-cell function [9]. However, it is easily degraded by dipeptidyl peptidase IV (DPP-IV), with a half-life less than $2 \mathrm{~min}$, which greatly limits its clinical application. This problem was overcome by the development of synthetic GLP-1 receptor agonists, such as exenatide (EX), which have been clinically used for the treatment of DM2, providing better glycemic control. In an openlabel prospective randomized research, 12 weeks of EX treatmet produced a significant weight loss and improved insulin resistance in overweight/obese women with PCOS compared with MF treatment [10]. This is in line with another study, which showed that EX appeared to be superior to MF in restoring menstrual cycles and regulating metabolic disorders [11]. However, its mechanism of improving IR has not yet been addressed in women with PCOS.

In our previous study [12, 13], insulin resistance in PCOS rats was associated with the AMPK $\alpha$-SIRT1 pathway. Therefore, in this study, we used MF or EX to intervene PCOS rats to compare their influences on metabolic abnormalities and to investigate whether their protective effects were related to the AMPK $\alpha$-SIRT1 pathway.

\section{Materials and methods PCOS rats models}

The Animal Experimental Center of Sun Yat-sen University Medical College (SCXK (GuangDong) 20110029) provided fifty female SD rats (25-day-old). These female rats were all specific-pathogen-free (SPF) grades with an average body weight of $79.79 \pm 4.18 \mathrm{~g}$. The rats were randomly divided into two groups: PCOS model group $(n=37)$ and normal control group $(n=13)$. The PCOS group rats were subcutaneously injected for 20 days with dehydroepiandrosterone (DHEA $6 \mathrm{mg} /(100$ $\mathrm{g} \cdot \mathrm{d})$ ) (Millipore (252805)) and $0.2 \mathrm{ml}$ injectable soybean oil; while the $\mathrm{NC}$ group rats were subcutaneously injected with only $0.2 \mathrm{ml}$ of injectable soybean oil. The rats' weight were recorded daily. After ten days of injections, the rats in both groups were vaginally swabbed daily and the discharge was observed under the microscope throughout three estrous cycles. After the estrous cycle of the PCOS group disappeared or irregular, the PCOS model were considered to have been successfully established. Eight rats were randomly selected (3 from the control group and 5 from the PCOS group) for the fasting blood glucose, serum testosterone and fasting insulin tests, as well as for histological examination of their ovarian issues to further evaluate the efficiency of model establishment.

The remaining 42 rats were randomly divided into 4 groups: MF group $(n=11)$, EX group $(n=11)$, PCOS group $(n=10)$ and the NC group (NC group, $\mathrm{n}=10)$. The MF group was administered MF $300 \mathrm{mg} /(\mathrm{kg} \bullet \mathrm{d})$ daily, dissolved in $0.2 \mathrm{ml}$ sterile distilled water. The EX group was subcutaneously injected EX $10 \mu \mathrm{g} /(\mathrm{kg} \bullet \mathrm{d})$ daily, dissolved in $0.2 \mathrm{ml}$ of sterile distilled water. The PCOS group and the $\mathrm{NC}$ group were subcutaneously injected with only $0.2 \mathrm{ml}$ of sterile distilled water every day. All injections lasted for 4 weeks (Fig. 1).

\section{Blood and ovarian tissue collection}

The blood and ovarian tissue collection and the hematoxylin-eosin (HE) staining was done as previously described [12]. In the microscopic examination, 5 fields were randomly selected in every pathological section for observation and the number of immature follicles was counted under high power microscope fields (HPF) (400X). A total of 10 sections were observed [14].

\section{Western blot assays (WB)}

The primary antibody was purchased from Cell Signaling Technology (CST): anti-AMPK $\alpha$ (5832S), anti-pAMPKa1/ 2 (2535S), anti-SIRT1 (8469S). All corresponding secondary antibodies were purchased from Sino Biological (China, Beijing).

\section{Quantitative real-time polymerase chain reaction (qPCR)}

Total RNA from ovarian tissues was extracted using Trizol reagent (Invitrogen), and cDNA was generated using a reverse transcription kit (Takara (RR047A)). The RT-PCR kit was purchased from Takara (RR820A). Primer sequences were as follows: AMPK $\alpha$ : 5'-TAAACC CACAGAAATCCAAACACC-3' (forward), 5' -ACAACC TTCCATTCATAGTCCAACT-3'(reverse); SIRT1: 5' AACCACCAAAGCGGAAAAAAAGAA-3'(forward), 5' CCACAGCAAGGCGAGCATAAATA-3'(reverse); endogenous control $\beta$-actin: 5'-CCGTAAAGACCTCT ATGCCAACA-3' (forward), 5'-CTAGGAGCCAGGGC AGTAATCTC-3' (reverse). 


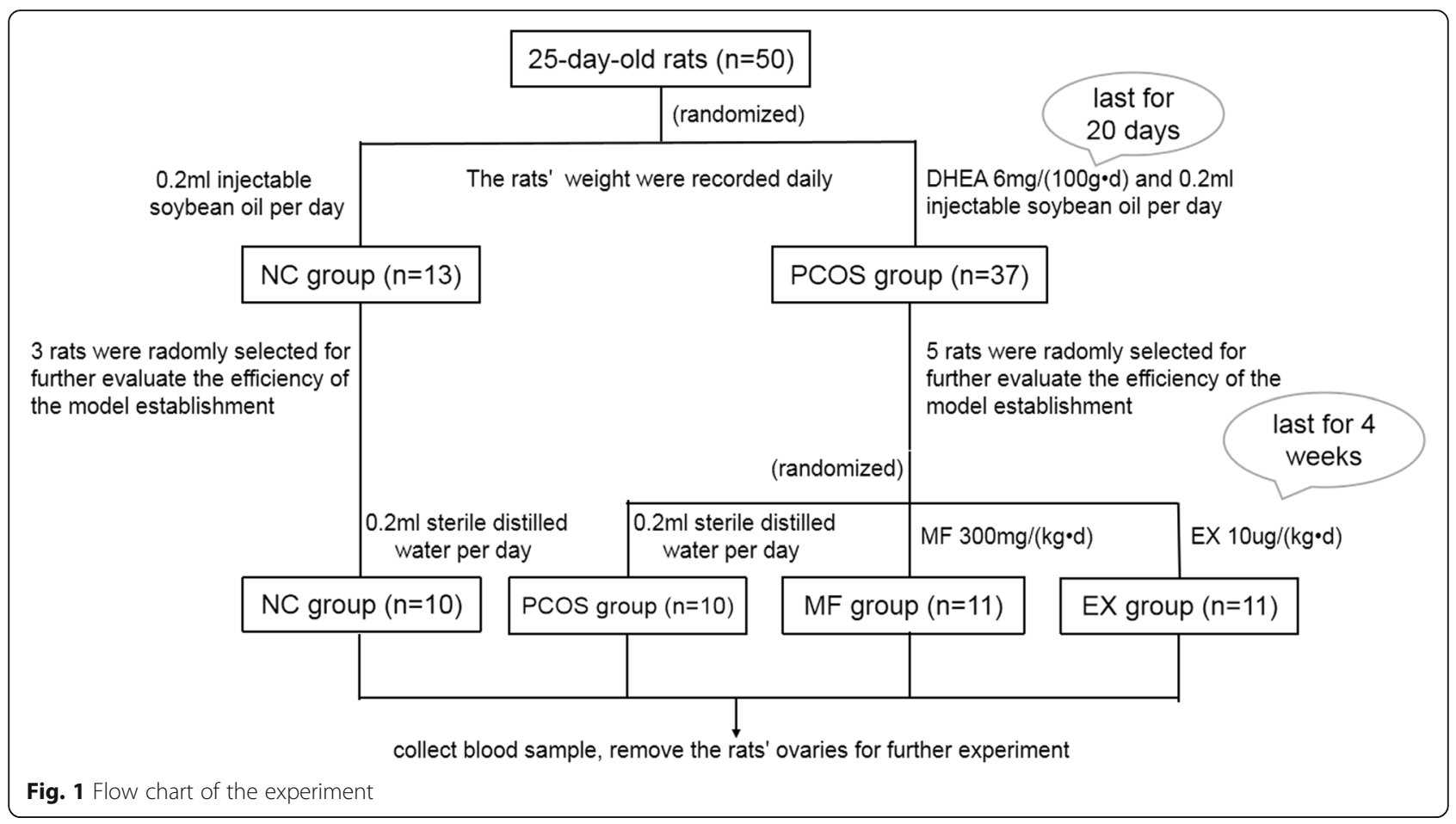

\section{Statistical analysis}

Data statistics and analysis were performed using SPSS 21.0 software (SPSS Inc., Chicago, IL, USA). The results were expressed in mean \pm standard deviation (SD) or median and interquartile ranges. An independent two samples $\mathrm{T}$ test was used for homogeneity of variance, otherwise the non-parametric test was used. One-way ANOVA was carried out when multiple comparisons were evaluated. The difference was considered statistically significant at $P<0.05$.

\section{Results}

\section{Estrous cycle monitoring and parameters of rats after} DHEA pretreatment

Rats in the PCOS group lost their regular estrous cycles and remained in the diestrus phase after DHEA treatment. Whereas the estrous cycle of the control group was still regular at about 4-5 days. As shown in the Table 1A, after 20 days of DHEA treatment, compared with those in the control group, body weights (166.38 \pm 7.69 vs $158.92 \pm 10.06 \mathrm{~g}, P=0.008)$ and fasting blood glucose (FBG) $(9.50 \pm 0.60$ vs $7.90 \pm 0.60 \mathrm{mmol} / \mathrm{L}, P=$ 0.01 ) in the PCOS group were increased significantly. Fasting insulin levels (FINS) in the PCOS group $(30.12 \pm 6.63$ vs $23.07 \pm 2.07 \mathrm{mU} / \mathrm{L}, P=0.132)$ were also higher than those in the control group, although the difference was not statistically significant. Furthermore, the PCOS group showed prominent hyperandrogenemia $(4.92 \pm 2.41$ vs $0.12 \pm 0.07 \mathrm{ng} / \mathrm{ml}, P=0.011)$ and IR (measured by HOMA-IR, $12.63 \pm 2.32$ vs $8.10 \pm 0.93, P=$
0.02), suggesting the successful establishment of PCOS rats.

Ovarian morphologic changes after DHEA pretreatment In the control group, follicles of different developmental stages and a few corpora lutea were observed. The granulosa cells were orderly arranged in an intact form, mostly in 4-6 layers. However, the number of immature follicles was significantly increased $(13.20 \pm 2.38$ vs $8.00 \pm 1.00, P=0.002)$ in the PCOS group, and the corona radiation of oocytes disappeared, and granulosa cells were arranged loosely in fewer (only 1-3) layers (Fig. 2, Table 2).

\section{Parameters of rats after metformin or exenatide intervention}

As shown in Table 1B, compared with the PCOS group, body weights $(222.64 \pm 16.57,218.63 \pm 13.18$ vs $238.30 \pm$ $12.26 \mathrm{~g}, P=0.026)$ and serum testosterone $(0.09 \pm 0.03$, $0.09 \pm 0.03$ vs $0.53 \pm 0.41 \mathrm{ng} / \mathrm{ml}, P<0.01)$ in the MF group and EX group were significantly decreased. Moreover, the insulin sensitivity of MF and EX groups had imrpoved $(P<0.01)$. The body weight $(218.63 \pm 13.18 \mathrm{vs}$ $222.64 \pm 16.57 \mathrm{~g})$ and HOMA-IR $(7.44 \pm 1.23$ vs $8.26 \pm$ 2.50) of the EX group were lower than those of the MF group, although the difference was not statistically significant. These results demonstrated that MF and EX both can improve metabolic abnormalities in PCOS rats. 
Table 1 Weight and serum hormone data

\begin{tabular}{|c|c|c|c|c|c|c|}
\hline Group & Weight(g) & FBG (mmol/L) & FINS (mU/L) & HOMA-IR & $\mathrm{T}(\mathrm{ng} / \mathrm{mL})$ & $\mathrm{LH}(\mathrm{m} / \mathrm{U} / \mathrm{L})$ \\
\hline \multicolumn{7}{|c|}{ A: Data and Comparison between Control group and PCOS group after continuous injection of DHEA for 20 days } \\
\hline Control $(n=3)$ & $158.92 \pm 10.06$ & $7.90 \pm 0.60$ & $23.07 \pm 2.07$ & $8.10 \pm 0.93$ & $0.12 \pm 0.07$ & $3.17 \pm 1.08$ \\
\hline $\operatorname{PCOS}(n=5)$ & $166.38 \pm 7.69$ & $9.50 \pm 0.60$ & $30.12 \pm 6.63$ & $12.63 \pm 2.32$ & $4.92 \pm 2.41$ & $3.64 \pm 1.50$ \\
\hline$P$ value/T test & $0.008^{*}$ & $0.01^{*}$ & 0.132 & $0.02^{*}$ & $0.011^{*}$ & 0.661 \\
\hline \multicolumn{7}{|c|}{ B: Data between 4 groups after continuous injection of metformin and exenatide for 4 weeks } \\
\hline$N C(n=10)$ & $222.60 \pm 17.88$ & $7.92 \pm 0.45$ & $23.38 \pm 3.24$ & $8.25 \pm 1.36$ & $0.08(0.06-0.10)$ & $3.02 \pm 0.73$ \\
\hline$P \operatorname{COS}(n=10)$ & $238.30 \pm 12.26^{\mathrm{a}}$ & $8.68 \pm 0.47^{\mathrm{a}}$ & $32.91 \pm 4.27^{\mathrm{a}}$ & $12.66 \pm 1.44^{\mathrm{a}}$ & $0.35(0.20-0.99)^{a}$ & $3.19 \pm 0.85$ \\
\hline$E X(n=11)$ & $218.63 \pm 13.18$ & $8.17 \pm 0.54$ & $20.51 \pm 3.53$ & $7.44 \pm 1.23$ & $0.08(0.08-0.10)$ & $2.99 \pm 0.57$ \\
\hline$M F(n=11)$ & $222.64 \pm 16.57$ & $7.71 \pm 0.72$ & $25.08 \pm 6.44$ & $8.26 \pm 2.50$ & $0.09(0.07-0.12)$ & $2.53 \pm 1.00$ \\
\hline$P$ value/one way ANOVA & $0.026^{*}$ & $<0.01^{*}$ & $<0.01^{*}$ & $<0.01^{*}$ & $<0.01^{*}$ & 0.276 \\
\hline
\end{tabular}

Mean Mean value, SD Standard Error, FBG Fasting blood Glucose, FINS Fasting insulin, $T$ Testosterone, HOMA-IR HOMA insulin Resistance index

*:P< 0.05 That means that the difference is statistically significant between groups

${ }^{a}$ : That means the difference between this group and other groups is statistically significant

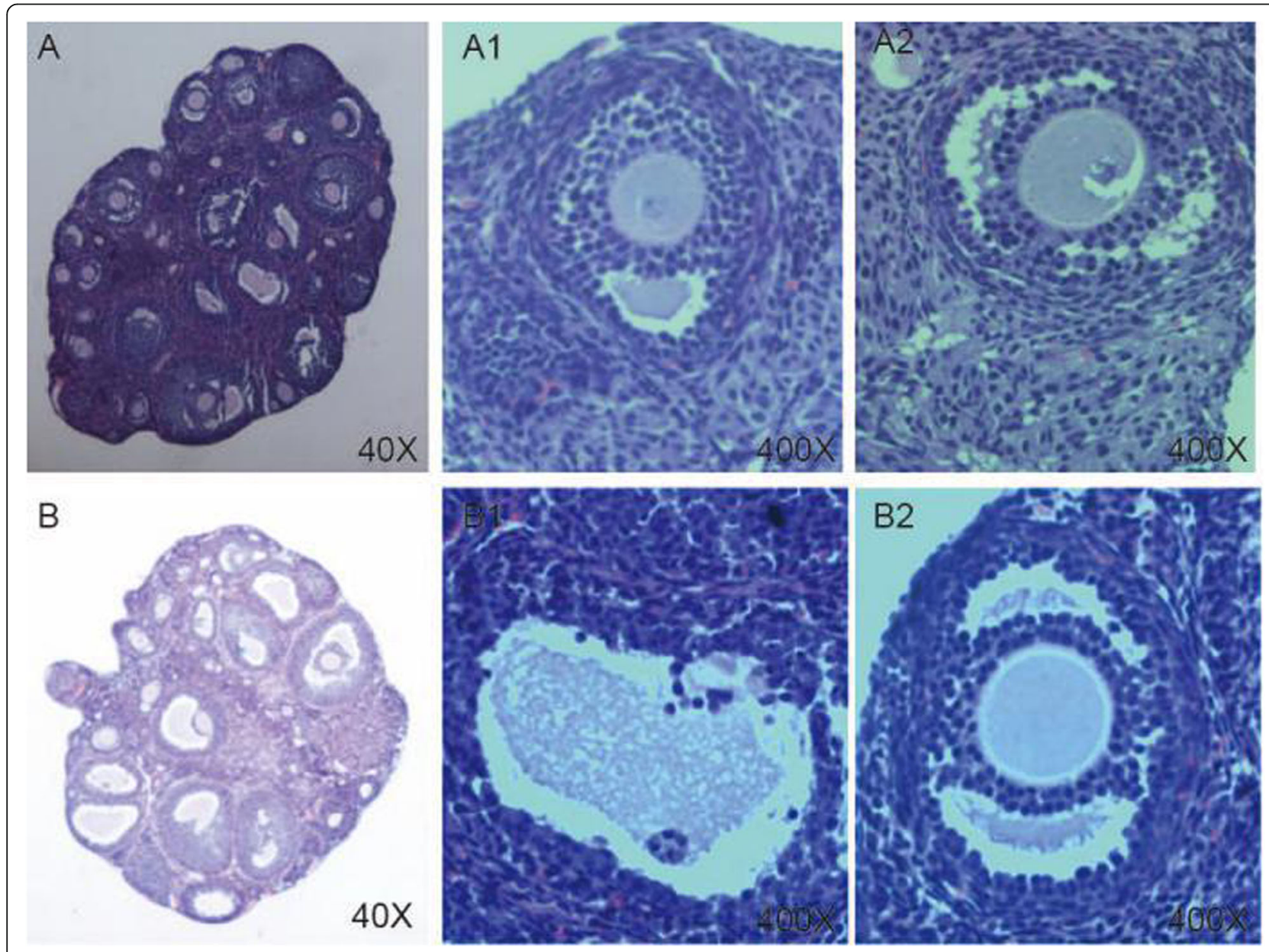

Fig. 2 HE staing of the ovaries of rats. a The ovaries of the control group rats (40X); A1, A2: Part of figure A (400X). b The ovaries of the PCOS group rats (40X); B1, B2: Part of figure B(400X) 
Table 2 Comparison of the number of immature small follicles between the two groups

\begin{tabular}{lll}
\hline Group & $\begin{array}{l}\text { The number of immature small } \\
\text { follicles /HPF }\end{array}$ & $P$ value/T test \\
\hline NC & $8.00 \pm 1.00$ & $0.002^{*}$ \\
PCOS & $13.20 \pm 2.38$ & $0.002^{*}$ \\
\hline
\end{tabular}

HPF High power field

${ }^{*} P<0.05$ That means the difference is statistically significant between groups

AMPKa and SIRT1 protein and mRNA expression in rat ovaries after metformin or exenatide intervention

DHEA treatment resulted in reduced expression of AMPK $\alpha$ protein, MF or EX treatment increased AMPK $\alpha$ protein expression. The SIRT1 expression was consistent with that of AMPK $\alpha$ in each group (Fig. 3a), suggesting that upregulation of the AMP $\alpha-S I R T 1$ molecular pathway can improve the IR status of PCOS rats.

The expression of AMPK $\alpha$ and SIRT1 mRNA were decreased in the PCOS group, while MF or EX treatment could increased the mRNA expression of AMPK $\alpha$ and
SIRT1, and restored the regular menstrual cycle. We conclude from the trend of expression that MF and EX may exert their protective effects on metabolic abnormalities in PCOS rats via AMPKa-SIRT1 pahtway (Fig. 3b).

\section{Discussion}

The clinical manifestation of PCOS is highly heterogeneous. It is a complex reproductive endocrine and psychological disease, which affects the health of women throughout their life [1]. PCOS is related to a series of reproductive, obstetrical, metabolic and psychological symptoms. The clinical manifestations of reproduction and obstetrics include menstrual disorder, hyperandrogenism, sterility and pregnancy concomitant symptoms, such as gestational diabetes mellitus, gestational hypertension, early abortion and neonatal concomitant symptoms [2]. Metabolic clinical manifestations include metabolic syndrome, IGT, type 2 diabetes, cardiovascular diseases and so on. Furthermore, PCOS patients are often accompanied by psychological symptoms, including depression and

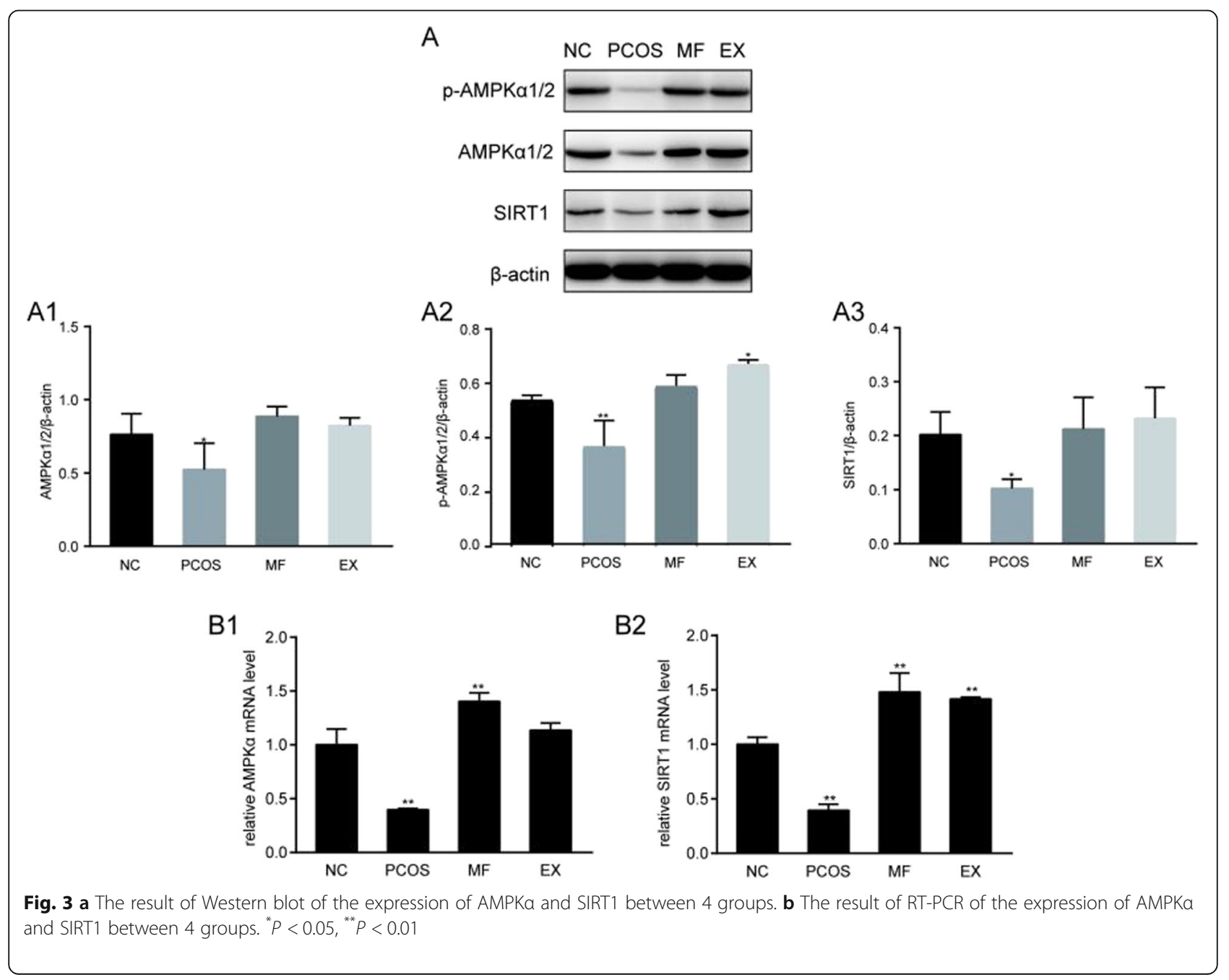


inferiority, which affect the quality of life [15]. In our study, rats in the PCOS group lost their regular estrous cycle, the microscopic examination revealed the presence of increased number of immature follicles. These results suggested that there were ovulatory disorders and ovarian polycystic changes in PCOS group, which is also an important clinical manifestation of PCOS. In addition, the body weight, serum testosterone and HOMA-IR in PCOS group were significantly higher than those in control group, suggesting that PCOS group was in an apparently IR status and accompanied by obesity, hyperandrogenism. IR seems to be an important determinant of metabolic disorders in patients with PCOS [16]. IR leads to increased insulin secretion from the pancreas to maintain normal blood glucose levels, resulting in compensated hyperinsulinemia, which in turn stimulates fat storage and affects cholesterol and lipoprotein metabolism. Besides, insulin can directly stimulate the activity of cytochrome P450c17 $\alpha$ enzyme in follicular membrane and promote the conversion of cholesterol to progesterone and progesterone to androgen. Insulin can also directly promote pituitary secretion of $\mathrm{LH}$, which acts on receptors on theca cells, further increasing androgen production [17]. On the other hand, abdominal obesity and elevated androgen also affect metabolic disorders, which in turn promote the production of insulin resistance. A recent meta analysis [18] used gold standard insulin clamp technique to evaluate the degree of insulin resistance in PCOS. The results showed that the insulin sensitivity of PCOS patients was $27 \%$ lower than that of the control group, and this had nothing to do with BMI, age or diagnostic criteria.

MF, an insulin sensitizer, has been introduced as a pharmaceutical option targeting not only IR, but also several other aspects of PCOS [4]. MF counteracts adipose tissue expansion by directly inhibiting lipogenesis. Culturing of pre-adipocytes in the presence of MF resulted in increased phosphorylation of AMPK at Thr172 and the accumulation of significantly less lipid than in non-treated cells [19]. This observation may be related to the potential weightloss favoring effect of MF. However, MF could not activate purified rat liver AMPK, indicating that it is not a direct activator of AMPK, and its activation of AMPK depends on the presence of intact cells. Shaw et al. [20] showed that the activity of liver AMPK disappeared if liver kinase B1 (LKB1), the AMPK upstream kinase, was knocked out, and MF also lost its hypoglycemic effect. Therefore, MF was thought to act through the LKB1-AMPK pathway.

In addition to its hypoglycaemia action, MF can also protect microvascular endothelial cells from glucose toxicity by a mechanism that may involves SIRT1-mediated growth arrest [21]. AMPK $\alpha$ elevates the expression of SIRT1 by up-regulating the intracellular levels of its cosubstrate NAD+ or the activity of nicotinamide [22]. Similarly, SIRT1 can activate AMPK via deacetylation of LKB1, which promotes LKB1 translocation from the nucleus to the cytosol, where it is activated and phosphorylates and activates AMPK [23]. A similar action of MF via the AMPK $\alpha$-SIRT1 pathway has also been shown in hepatic HepG2 cells under high glucose conditions [24]. This finding is consistent with our study that the levels of AMPK $\alpha$ and SIRT1 in the ovary of PCOS rats were significantly lower than those in the control group. The expressions of AMPK $\alpha$ and SIRT1 were significantly increased after AMPK $\alpha$ agonists treatment, such as MF.

Although MF has been widely used to improve IR in patients with PCOS, many patients can not tolerate its gastrointestinal side effects, and its weight control effect is not satisfactory [7]. In our study, GLP-1 receptor agonists EX and MF significantly improved insulin resistance and endocrine disorder in PCOS, and the average body weight and HOMA-IR of rats in EX group were lower than those in MF group, although the difference was not statistically significant. This may be related to the short duration of intervention. Our results are consistent with the results of a non-blind prospective randomized controlled study [10] of obese PCOS patients. In that study, the experimental group was treated with subcutaneous EX (10 $\mu \mathrm{g}$ bid) for 12 weeks, while the control group was given oral MF (1000 mg bid). The result showed that EX group had more significant weight loss and improved HOMA-IR, and the natural pregnancy rate of EX group was higher than that of MF group. GLP-1, a potent antidiabetic incretin hormone produced by intestinal cells, is widely used for DM2 treatment because of its action to stimulate insulin secretion, suppress glucagon production and release in a glucose-dependent manner. Despite its potent insulinotropic effect, the clinical application of oral GLP-1 is greatly limited by its instability in the gastrointestinal tract, poor absorption efficiency and rapid degradation by DPP4 [25]. Various GLP-1 receptor agonists, such as $\mathrm{EX}$, have been developed to provide prolonged in vivo actions. EX, with a half-life of more than $2.4 \mathrm{~h}$, only increases insulin release in the case of hyperglycaemia and therefore does not cause hypoglycaemia [8]. EX decreases glucagon release after binding to the its receptor (GLP-1R) present on pancreatic endocrine $\alpha$ - and $\beta$ cells [26]. GLP-1R is coupled to G protein, which, once activated, increases intracellular cyclic AMP (cAMP) and induces extracellular signal-regulated kinase (ERK) $1 / 2$, protein kinase A (PKA) and phosphoinositol 3 Activation of kinase (PI3K)/protein kinase B (PKB) [27].

Obesity, insulin resistance and hyperandrogenism are often associated with PCOS, improved weight control and 
glycemic profiles often result in prevention of metabolic syndrome in women with PCOS [16]. In the present study, we found that after 4 weeks of MF or EX treatment, body weight, fasting blood glucose and HOMA-IR were significantly reduced compared with the untreated PCOS group. In addition, after MF or EX treatment, the elevation in AMPK $\alpha$ and SIRT1 expression indicated that AMPK $\alpha-$ SIRT1 pathway might participate in the improvement of metabolic disorder due to MF or EX treatment. Nevertheless, whether the effects of GLP-1 are mediated via the activation of SIRT1 and/or directly via AMPK still requires further studies.

\section{Conclusion}

In conclusion, MF and GLP-1 receptor agonists, such as EX, can significantly improve insulin resistance in PCOS rats, and their action may be in relation to the AMPK $\alpha$ SIRT1 pathway. Therefore, the AMPK $\alpha$-SIRT1 pathway is expected to be an important target for the treatment of patients with PCOS. This matter deserves further attention. Larger trials are needed to explore the mechanism of EX in reducing body weight and improving IR in women with PCOS.

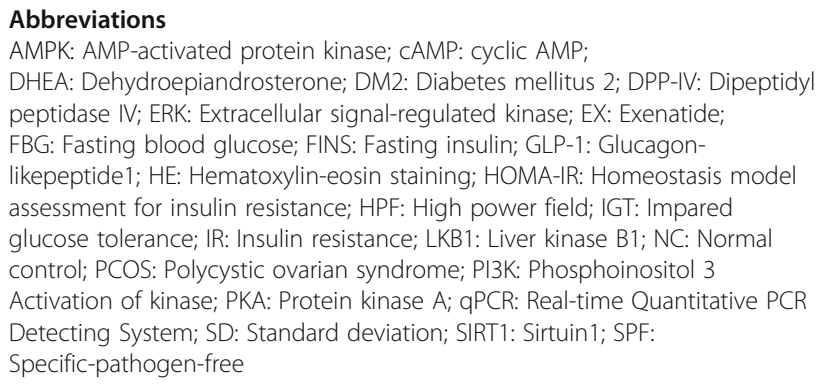

\section{Acknowledgements}

The authors would like to acknowledge Professor Mike for his help with the manuscript.

\section{Authors' contributions}

CL carried out establishment of PCOS model. GSQ carried out the blood and ovarian tissue collection. DXY participated in Western and qPCR. CLS participated in the design of the study and performed the statistical analysis. TX conceived of the study, and approved the final manuscript.

\section{Funding}

This research was funded by an internal fund of Sun Yat-sun University.

\section{Availability of data and materials}

Please contact author for data requests.

\section{Ethics approval and consent to participate}

All procedures involving rats were carried out in accordance with the strict standards of the National Institutes of Health guide for the care and use of laboratory animals, and the program was approved by the Institutional Animal Care and Use Commitee of Sun Yat-sen University.

\section{Consent for publication}

Not applicable.

\section{Competing interests}

The authors declare that they have no competing interests.

\section{Author details}

'Center for Reproductive Medicine, The Third Affiliated Hospital, Sun Yat-sen University, 600, Thianhe Road, Guangzhou, Guangdong, People's Republic of China 510630. ${ }^{2}$ Ultrasound Department, The First Affiliated Hospital, Sun Yat-sen University, Guangzhou, Guangdong, People's Republic of China 510080. ${ }^{3}$ Center for Republic Medicine, The Sixth Affiliated Hospital of GuangZhou Medical University, The People's Hospital of Qingyuan,

Qingyuan, Guangdong, People's Republic of China 511500.

Received: 21 February 2019 Accepted: 15 August 2019

Published online: 16 September 2019

\section{References}

1. Moran L, Teede H. Metabolic features of the reproductive phenotypes of polycystic ovary syndrome. Hum Reprod Update. 2009;15:477-88. https:// doi.org/10.1093/humupd/dmp008.

2. Hirschberg AL. Polycystic ovary syndrome, obesity and reproductive implications. Women Health. 2009;5:529-42. https://doi.org/10.2217/whe.09.39.

3. Cooney LG, Dokras A. Beyond fertility: polycystic ovary syndrome and longterm health. Fertil Steril. 2018;110:794-809. https://doi.org/10.1016/j. fertnstert.2018.08.021.

4. Diamanti-Kandarakis E, Christakou CD, Kandaraki E, Economou FN. Metformin: an old medication of new fashion: evolving new molecular mechanisms and clinical implications in polycystic ovary syndrome. Eur J Endocrinol. 2010;162: 193-212. https://doi.org/10.1530/EJE-09-0733.

5. Velazquez EM, Mendoza S, Hamer T, Sosa F, Glueck CJ. Metformin therapy in polycystic ovary syndrome reduces hyperinsulinemia, insulin resistance, hyperandrogenemia, and systolic blood pressure, while facilitating normal menses and pregnancy. Metabolism. 1994;43:647-54. https://doi.org/10.1 016/0026-0495(94)90209-7.

6. Kim YD, Park K, Lee Y, Park Y, Kim D, Nedumaran B, et al. AMP-activated protein kinase - dependent regulation of the orphan nuclear receptor SHP. Diabetes. 2008;57:306-14. https://doi.org/10.2337/db07-0381.Additional.

7. Lyndal RH, Naveed S, Jane EN, Richard F. Metformin and weight loss in obese women with polycystic ovary syndrome: comparison of doses. J Clin Endocrinol Metab. 2005;90:4593-8. https://doi.org/10.1210/jc.2004-2283.

8. Bell Gl, Sanchez-Pescador R, Laybourn PJ, Najarian RC. Exon duplication and divergence in the human preproglucagon gene. Nature. 1983;304:368-71. https://doi.org/10.1038/304368a0.

9. Meier JJ. GLP-1 receptor agonists for individualized treatment of type 2 diabetes mellitus. Nat Rev Endocrinol. 2012;8:728-42. https://doi.org/10.103 8/nrendo.2012.140.

10. Liu X, Zhang Y, Zheng SY, Lin R, Xie YJ, Chen $\mathrm{H}$, et al. Efficacy of exenatide on weight loss, metabolic parameters and pregnancy in overweight/obese polycystic ovary syndrome. Clin Endocrinol (Oxf). 2017;87(6):767-74. https:// doi.org/10.1111/cen.13454

11. Elkind-Hirsch $\mathrm{K}$, Marrioneaux $\mathrm{O}$, Bhushan $\mathrm{M}$, Vernor D, Bhushan $\mathrm{R}$. Comparison of single and combined treatment with exenatide and metformin on menstrual cyclicity in overweight women with polycystic ovary syndrome. J Clin Endocrinol Metab. 2008;93(7):2670-8. https://doi. org/10.1210/jc.2008-0115.

12. Tao X, Chen L, Cai L, Ge S, Deng X. Regulatory effects of the AMPKa-SIRT1 molecular pathway on insulin resistance in PCOS mice: an in vitro and in vivo study. Biochem Biophys Res Commun. 2017;494:615-20. https://doi. org/10.1016/j.bbrc.2017.09.154.

13. Tao $X$, Zhang $X$, Ge SQ, Zhang EH, Zhang B. Expression of SIRT1 in the ovaries of rats with polycystic ovary syndrome before and after therapeutic intervention with exenatide. Int J Clin Exp Pathol. 2015;8(7):8276-83.

14. Gougeon A. Dynamics of follicular growth in the human: a model from preliminary results. Hum Reprod. 1986;2:81-7. https://doi.org/10.1093/ oxfordjournals.humrep.a136365.

15. Veltman-Verhulst SM, Boivin J, et al. Emotional distress is a common risk in women with polycystic ovary syndrome: a systematic review and metaanalysis of 28 studies. Hum Reprod Update. 2012;18:638-51. https://doi. org/10.1093/humupd/dms029.

16. Neven ACH, Laven J, Teede HJ, Boyle JA. A summary on polycystic ovary syndrome: diagnostic criteria, prevalence, clinical manifestations, and management according to the latest international guidelines. Semin Reprod Med. 2018;36:5-12. https://doi.org/10.1055/s-0038-1668085.

17. Diamanti-Kandarakis E, Papavassiliou AG. Molecular mechanisms of insulin resistance in polycystic ovary syndrome. Trends Mol Med. 2006;12:324-32. 
18. Cassar S, Misso ML, Hopkins WG, et al. Insulin resistance in polycystic ovary syndrome: a systematic review and meta-analysis of euglycaemichyperinsulinaemic clamp studies. Hum Reprod. 2016;31:2619-31. https://doi. org/10.1093/humrep/dew243.

19. Tobergte $D R$, Curtis $S$. Metformin inhibits intra-cellular lipid accumulation in the murine preadipocyte cell line, 3T3-L1. J Chem Inf Model. 2013;53:1689-99. https:/doi.org/10.1017/CBO9781107415324.004

20. Shaw RJ, Lamia KA, Vasquez D, Koo SH, Bardeesy N, DePinho RA, et al. Medicine: the kinase LKB1 mediates glucose homeostasis in liver and therapeutic effects of metformin. Science (80- ). 2005;310:1642-6. https:// doi.org/10.1126/science.1120781.

21. Arunachalam G, Samuel SM, Marei I, Ding H, Triggle CR. Metformin modulates hyperglycaemia-induced endothelial senescence and apoptosis through SIRT1. Br J Pharmacol. 2014;171:523-35. https://doi.org/10.1111/bph.12496.

22. Canto' C, Gerhart-Hines Z, Feige JN, et al. AMPK regulates energy expenditure by modulating NAD+ metabolism and SIRT1 activity. Nature. 2009:458:1056-60. https://doi.org/10.1038/nature07813.

23. Rogacka D, Audzeyenka I, Rychłowski M, Rachubik P, Szrejder M, Angielski S, et al. Metformin overcomes high glucose-induced insulin resistance of podocytes by pleiotropic effects on SIRT1 and AMPK. Biochim Biophys Acta - Mol Basis Dis. 2018;1864:115-25. https:/doi.org/10.1016/.j.bbadis.2017.10.014.

24. Nelson LE, Valentine RJ, Cacicedo JM, Gauthier M-S, Ido Y, Ruderman NB. A novel inverse relationship between metformin-triggered AMPK-SIRT1 signaling and p53 protein abundance in high glucose-exposed HepG2 cells. Am J Physiol Physiol. 2012;303:C4-13. https://doi.org/10.1152/ajpcell.00296.2011.

25. Shrestha N, Araújo F, Shahbazi MA, Mäkilä E, Gomes MJ, Airavaara M, et al. Oral hypoglycaemic effect of GLP-1 and DPP4 inhibitor based nanocomposites in a diabetic animal model. J Control Release. 2016;232: 113-9. https://doi.org/10.1016/j.jconrel.2016.04.024.

26. Andreozzi F, Raciti GA, Nigro C, Mannino GC, Procopio T, Davalli AM, et al. The GLP-1 receptor agonists exenatide and liraglutide activate glucose transport by an AMPK-dependent mechanism. J Transl Med. 2016;14:1-13. https://doi.org/10.1186/s12967-016-0985-7.

27. Thorens B. Expression cloning of the pancreatic J8 cell receptor for the gluco-incretin hormone glucagon-like peptide 1 (insulin secretion/noninsulin-dependent diabetes mellitus/entero-insular axis/G proteins/cAMP). Cell Biol. 1992;89:8641-5.

\section{Publisher's Note}

Springer Nature remains neutral with regard to jurisdictional claims in published maps and institutional affiliations.

Ready to submit your research? Choose BMC and benefit from:

- fast, convenient online submission

- thorough peer review by experienced researchers in your field

- rapid publication on acceptance

- support for research data, including large and complex data types

- gold Open Access which fosters wider collaboration and increased citations

- maximum visibility for your research: over $100 \mathrm{M}$ website views per year

At $\mathrm{BMC}$, research is always in progress.

Learn more biomedcentral.com/submissions 